\title{
GUTACHTER(INNEN) 2012
}

Die Redaktion der PVS dankt allen Gutachterinnen und Gutachtern, die ihr bei der Auswahl der Beiträge im Jahr 2012 behilflich waren und mit ihrem Engagement bei der sorgfältigen und konstruktiven Prüfung der eingegangenen Manuskripte wesentlich zur Sicherung der Qualität der PVS beigetragen haben.

Mathias Albert
Kai Arzheimer
Christine Bauhardt
Timm Beichelt
Hans-Jürgen Bieling
Dietmar Braun
André Brodocz
Hauke Brunkhorst
Marius Busemeyer
Sergio Costa
Sabine Demel
Andreas Dür
Martin Elff
Ulf Engel
Franz Fallend
Simon Franzmann
Brigitte Geißel
Florian Grotz
Christoph Haas
Silja Häusermann
Andreas Hetzel
Achim Hurrelmann
Anja Jetschke
Dirk Jörke

Simon Joss

Uwe Jun

Achim Kemmerling

Sigrid Koch-Baumgarten

Michael Kolkmann

Regina Kreide

Sandra Kröger

Sabine Kropp

Andreas Ladner

Bern Ladwig

Simone Leiber

Dirk Leuffen

Sven Leunig

Marcus Maurer

Walter Müller

Andreas Niederberger

Frank Nullmeier

Franz Urban Pappi

Fabian Pfeffer

Sven-Oliver Proksch

Christian Rauh

Carsten Reinemann

Henrik Scheller

Klaus Schlichte
Manfred G. Schmidt

Carina Schmitt

Kai-Uwe Schnapp

Volker Schneider

Daniel Schulz

Siegfried Schumann

Peter Selb

Markus Steinbrecher

Volker Stocké

David Strecker

Gerd Strohmeier

Roland Sturm

Ingeborg Tömmel

Carsten Ullrich

Kerstin Völkl

Christoph Weller

Claus Wendt

Bernhard Weßels

Antje Wiener

Frieder Wolf

Thomas Zittel

Christoph Zürcher 